\title{
ROR1 is a pseudokinase that is crucial for MET- driven tumorigenesis
}

\author{
Luca Lazzari ${ }^{1,3^{*}}$, Alessandra Gentile ${ }^{1}$, Silvia Benvenuti ${ }^{1}$, Livio Trusolino ${ }^{2,3}$, Paolo M Comoglio ${ }^{1,3}$ \\ From São Paulo Advanced School of Comparative Oncology \\ Águas de São Pedro, Brazil. 30 September - 6 October 2012
}

\section{Background}

Aberrant deregulation of some Receptor Tyrosine Kinases (RTKs) signalling underlies diverse facets of tumor pathobiology providing an attractive target for cancer therapy. Searching for novel cancer-associated RTKs is an important issue to discover new therapeutic opportunities. For this reason, we investigated the contribution of Receptor tyrosine kinase-like Orphan Receptor 1 (ROR1) to human cancer.

\section{Patients and methods}

The level of ROR1 protein expression, phosphorylation and cellular growth response to RNAi-mediated ROR1 knockdown was evaluated by an integrated screening in a panel of 43 cancer cell lines. ROR1 auto-kinase activity and transphosphorylation were determined by biochemical assays. Functional consequences of ROR1 silencing were evaluated by several in vitro and in vivo biological assays.

\section{Results}

We demonstrated that although ROR1 is expressed in approximately $75 \%$ of the screened cancer cell lines, only gastric carcinoma cells (HS746T) and non-small cell lung carcinoma cells (NCI-H1993) exhibit high levels of ROR1 tyrosine phosphorylation and experience growth inhibition upon ROR1 suppression. Biochemical assays revealed that ROR1 is a pseudokinase lacking autocatalytic activity. Intriguingly, the two phospho-ROR1 positive cell lines both exhibited amplification and constitutive activation of the $M E T$ oncogene. ROR1 phosphorylation was abrogated by MET inhibition, indicating MET dependent transphosphorylation of ROR1. Silencing of
ROR1 in HS746T and NCI-H1993 cells impaired cellular proliferation, growth and migration in vitro and induced a dramatic inhibition of tumorigenesis in vivo.

\section{Conclusions}

Our data show that ROR1 is a pseudokinase functionally transphosphorylated by MET RTK, suggesting a critical role for ROR1 in malignant phenotypes sustained by the MET oncogene.

\section{Financial support}

AIRC special program Molecular Clinical Oncology "5x1000" and AIRC grants.

\section{Author details}

'Exploratory Research Laboratory, Institute for Cancer Research and Treatment (IRCC), Candiolo (TO), Italy. ${ }^{2}$ Molecular Pharmacology Laboratory, Institute for Cancer Research and Treatment (IRCC), Candiolo (TO), Italy. ${ }^{3}$ Department of Oncological Sciences, University of Turin Medical School, Candiolo (TO), Italy.

Published: 4 April 2013

doi:10.1186/1753-6561-7-S2-P43

Cite this article as: Lazzari et al:: ROR1 is a pseudokinase that is crucial for MET-driven tumorigenesis. BMC Proceedings 2013 7(Suppl 2):P43.

\footnotetext{
* Correspondence: luca.lazzari@ircc.it

'Exploratory Research Laboratory, Institute for Cancer Research and Treatment (IRCC), Candiolo (TO), Italy

Full list of author information is available at the end of the article
}

(c) 2013 Lazzari et al; licensee BioMed Central Ltd. This is an Open Access article distributed under the terms of the Creative Commons 